\title{
1 Bone morphogenetic protein 9 induces osteogenic differentiation of germ cell 1
}

\section{2 spermatogonial cells}

3 Short Title: BMP-9 in spermatogonial cell differentiation

4 Jiye Zhang ${ }^{\mathrm{a}, \mathrm{b}}$, Bangfa $\mathrm{Xu}^{\mathrm{a}}$, Xinping Chen ${ }^{\mathrm{a}}$, Liqiang Zhaoc, Pei Zhang ${ }^{\mathrm{a}}$, Fei Wang ${ }^{\mathrm{d}}$, Xiaojuan

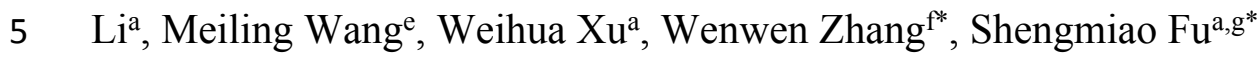

$7 \quad{ }^{a}$ Central Laboratory for Clinical Medicine and Medical Research, Hainan General Hospital,

$8 \quad$ Haikou 570311, Hainan Province, China

$9{ }^{b}$ Affiliated Hainan Hospital of Hainan Medical University, Haikou 570311, Hainan Province,

10 China

11 'Reproductive Medical Center, The Maternity and Child Health Hospital of Hainan Province,

12 Haikou 570311, Hainan Province, China

${ }^{d}$ Department of Urology, Hainan General Hospital, Haikou 570311, Hainan Province, China

eDepartment of Transplant Surgeons, Hainan General Hospital, Haikou 570311, Hainan

Province, China

16 Stem Cell Research Laboratory, Department of Obstetrics and Gynecology, Affiliated

17 University-Town Hospital, Chongqing Medical University, Chongqing 401331, Chongqing

18 City, China

${ }^{g}$ Key Laboratory for Cell and Molecular Genetic Translational Medicine of Hainan Province, 
*Corresponding authors: Wenwen Zhang, Stem Cell Research Laboratory, Department of 187254894@qq.com. Shengmiao Fu, Central Laboratory for Clinical Medicine and Medical Research, Hainan General Hospital, Haikou 570311, Hainan Province, China. Tel: 860898 6862 2590, email: smfu2000@126.com.

\section{Abstract}

Germ cell 1 spermatogonial (GC-1spg) cells are multipotent progenitor cells. We previously confirmed that bone morphogenetic protein (BMP) 9 is among the most osteogenic BMPs. However, whether GC-1spg cells are driven toward osteogenic differentiation under proper stimuli is uncertain. Additionally, the molecular mechanism of BMP9 remains unclear. In the present study, we aimed to determine whether BMP9 can induce osteogenic differentiation of GC-1spg cells. Recombinant adenoviruses were generated by the AdEasy system to regulate the BMP9 expression in GC-1spg cells. We identified osteogenic markers by real-time PCR and staining techniques in vitro. Ectopic ossification assays and histological analysis were also performed to verify the in vivo activity of BMP9. Finally, potential signaling pathways of BMP9 were assessed by transcriptome sequencing and KEGG enrichment analysis. Using recombinant adenoviruses, we demonstrate that BMP9 upregulates osteogenic markers including Runx2, osteocalcin, osteopontin, and Sox9. BMP9 also activates alkaline phosphatase activity and calcium deposition in GC-1spg cells. In vivo results show that BMP9 overexpression in GC-1spg cells promotes ectopic bone formation and chondrogenesis. In addition, RNA-sequencing and KEGG pathway analysis 
demonstrate that several signaling pathways are involved in BMP9-mediated osteogenesis. GC-1spg cells not only maintain spermatogenesis but also retain the ability to form bone tissue. Therefore, BMP9 activity in GC-1spg cells may help identify signaling pathways implicated in bone formation and could be of use in regenerative medicine.

Keywords: Bone morphogenetic protein 9, RNA-seq, KEGG pathway analysis, recombinant adenovirus, chondrogenesis

\section{Introduction}

Germ cell 1 spermatogonial (GC-1spg) cells are mouse spermatogonia that are immortalized by the SV40 large T antigen [1]. These cells can differentiate into diverse cell types or undergo self-renewal, depending on different factors $[2,3,4]$. GC-1spg cells are largely involved in spermatogenesis and have mesenchymal stem cell (MSC) differentiation potential $[5,6,7,8]$. In other words, GC-1spg cells can differentiate into different cell types, including those of the osteogenic, chondrogenic, or adipogenic lineages $[9,10,11,12]$. However, several molecular events $[13,14]$ and factors are involved in osteogenic differentiation of MSCs. Importantly, bone morphogenetic proteins (BMPs) are essential for this process $[15,16,17]$.

BMPs, members of the TGF- $\beta$ superfamily, are required for bone formation, stem cell differentiation, and male reproduction $[15,16,18,19,20]$. Thus far, more than 15 different BMPs have been identified. Our previous work showed that BMP9 is the most osteogenic BMP $[21,22,23,24]$. BMP9, also known as growth differentiation factor 2 (GDF-2)[25], was

67 originally isolated from the fetal mouse liver $[25,26]$. Bone formation involves BMP9 acting through the Notch signaling pathway [14,24,27]. Several metabolic processes are regulated 
by BMP9, including glucose and lipid metabolism [28,29], iron metabolism [30], endothelial function, and angiogenesis [31,32,33]. Accordingly, abnormal BMP9 expression may be involved in various diseases $[29,34,35,36]$.

Studies confirm that GC-1spg cells have MSC characteristics [4,7,12,37], however, few reports have focused on the BMP9-mediated osteogenic effect on GC-1spg cells. Indeed, MSCs can be isolated from the testis [5,6]. We analyzed how GC-1spg cells react to BMP9 and demonstrate that BMP9 can induce bone formation in GC-1spg cells both in vitro and in vivo $[10,17,38]$. In addition, we analyzed the GC-1spg cell transcriptome after BMP9 treatment [27] and investigated potential BMP9 signaling pathways by KEGG enrichment analysis.

\section{Materials and Methods}

\section{Cell culture and chemicals}

GC-1spg and HEK-293 cells were purchased from the American Type Culture Collection (ATCC). Cells were cultured in complete Dulbecco's Modified Eagle Medium (DMEM, HyClone, Logan, UT, USA) supplemented with 10\% fetal bovine serum (FBS, HyClone), $100 \mathrm{U} / \mathrm{ml}$ penicillin, and $100 \mathrm{~g} / \mathrm{ml}$ streptomycin. Cultured cells were incubated at $37^{\circ} \mathrm{C}$ in $5 \% \mathrm{CO}_{2}[1,39,40,41]$. Unless otherwise specified, all chemicals were purchased from SigmaAldrich (St. Louis, MO, USA) or Thermo Fisher Scientific (Waltham, MA, USA).

\section{Recombinant adenovirus construction}

To regulate BMP9 expression, recombinant adenoviruses were generated by the AdEasy system $[22,39,42,43]$. Human BMP9 DNA was amplified by high-fidelity PCR as follows: 96 ${ }^{\circ} \mathrm{C}$ for $45 \mathrm{~s}$, followed by 18 cycles at $92^{\circ} \mathrm{C}$ for $20 \mathrm{~s}, 55^{\circ} \mathrm{C}$ for $30 \mathrm{~s}$, and $70{ }^{\circ} \mathrm{C}$ for $45 \mathrm{~s}$, and a 
91 final $70^{\circ} \mathrm{C}$ incubation for $5 \mathrm{~min}$. The amplified sequence was cloned into the adenoviral

92 shuttle vector, which was transformed into HEK-293 cells to generate recombinant

93 adenoviruses [39]. The resultant adenoviruses (Ad-BMP9) also expressed green fluorescent

94 protein (GFP). Control adenoviruses expressing only GFP (Ad-GFP) were also constructed

$95[17,38]$.

\section{$R N A$ isolation, reverse transcription, and real-time PCR}

\begin{tabular}{c|c|c|c}
\hline Name & Primer & Sequence & Size \\
& & & \\
\hline \multirow{3}{*}{ Hum BMP9 } & Forward & 5'-CTTCCCATTGGCTGATGACG-3' & \multirow{2}{*}{ 189bp } \\
\cline { 2 - 3 } & & & \\
& Reverse & 5'-GCCACACTCATCCCCTCATA-3' & \\
\hline
\end{tabular}




\begin{tabular}{|c|c|c|c|}
\hline \multirow{2}{*}{ Mus GAPDH } & Forward & 5'-ATGGGTGTGAACCACGAGA-3' & \multirow{2}{*}{$229 b p$} \\
\hline & Reverse & 5'-CAGGGATGATGTTCTGGGCA-3' & \\
\hline \multirow{3}{*}{ Mus RUNX2 } & Forward & 5'-AGATGGGACTGTGGTTACCG-3' & \multirow{3}{*}{$203 b p$} \\
\hline & & & \\
\hline & Reverse & 5'-TAGCTCTGTGGTAAGTGGCC-3' & \\
\hline \multirow{3}{*}{ Mus Osteocalcin } & Forward & 5'-AGGACCCTCTCTCTGCTCA-3' & \multirow{3}{*}{$223 \mathrm{bp}$} \\
\hline & & & \\
\hline & Reverse & 5'-CGTCACAAGCAGGGTTAAGC-3' & \\
\hline \multirow{3}{*}{ Mus Osteopontin } & Forward & 5'-TCCAATCGTCCCTACAGTCG-3' & \multirow{3}{*}{$249 \mathrm{bp}$} \\
\hline & & & \\
\hline & Reverse & 5'-AGCTGACTTGACTCATGGCT-3' & \\
\hline \multirow{3}{*}{ Mus SOX9 } & Forward & 5'-ATGAAGATGACCGACGAGCA-3' & \multirow{3}{*}{$196 b p$} \\
\hline & & & \\
\hline & Reverse & 5'-TGCACACGGGGAACTTATCT-3' & \\
\hline \multirow{3}{*}{ Mus BMP9 } & Forward & 5'-CTTCCCATTGGCTGATGACG-3' & \multirow{3}{*}{$189 \mathrm{bp}$} \\
\hline & & & \\
\hline & Reverse & 5'-GCCACACTCATCCCCTCATA-3' & \\
\hline
\end{tabular}

112 ALP activity was assayed by histochemical staining [17,38,48,49,50]. Log-phase GC-1spg cells were seeded in SlideFlasks (Thermo Fisher Scientific) and infected with Ad-BMP9 or

114 Ad-GFP. At the indicated time points, ALP activity was assessed using a BCIP/NBT Chromogen assay kit (Solarbio, Beijing, China). Assays were repeated at least three times. 
117 Alizarin Red S staining was used to identify mineralized matrix nodules or calcium precipitation [17,38,51]. GC-1spg cells were seeded in SlideFlasks, infected with Ad-BMP9 or Ad-GFP adenoviruses, and maintained in DMEM containing ascorbic acid $(50 \mathrm{mg} / \mathrm{mL})$ and $\beta$-glycerophosphate (10 mM) for 7 or 9 days [51]. Culture medium was then discarded, and the cells were carefully washed thrice with PBS. Cells were then fixed with $1 \%$ glutaraldehyde at room temperature for $10 \mathrm{~min}$ and rinsed thrice with PBS. Next, cells were incubated for $30 \mathrm{~min}$ at approximately $28{ }^{\circ} \mathrm{C}$ in $0.2 \%$ Alizarin Red S (Solarbio) and rinsed thrice with PBS. Calcium mineral deposits were observed with bright field microscopy.

\section{Cell implantation and ectopic ossification assay}

126

127

Animal experiments were approved and supervised by the research ethics committee of Hainan General Hospital. Ectopic bone formation assays were performed as previously described [17,38,52,53,54]. First, sub-confluent GC-1spg cells were treated with Ad-BMP9 or Ad-GFP for $24 \mathrm{~h}$. Cells were harvested $\left(5 \times 10^{6}\right.$ cells per sample) and resuspended in 100 $\mu \mathrm{L}$ PBS. Transformed cells were subcutaneously injected into the flanks of 4-week-old male athymic nude mice (5 animals per group). After 4 weeks, mice were euthanized, and injection-site tissue was collected for further analysis. Characteristic masses were selected, fixed in 10\% buffered formalin, and imaged using a micro-CT system (Skyscan 1076, Antwerp, Belgium). Tissue volume, bone volume, and bone surface area were measured for each tissue sample $[55,56]$.

\section{Histological analysis}

Collected tissue samples were fixed in $10 \%$ buffered formalin overnight and embedded in 
paraffin. Serial sections were stained with hematoxylin and eosin (H\&E, Sigma-Aldrich), Masson's trichrome (Sigma-Aldrich), and alcian blue (Sigma-Aldrich) [17,27,38,53].

\section{Signaling pathway analysis}

Transcriptome sequencing and KEGG enrichment analysis were performed for BMP9 relevant signaling pathway analysis. GC-1 spg cells were seeded in $100 \mathrm{~mm}$ culture dishes and infected with Ad-BMP9 or Ad-GFP. Total RNA was collected $48 \mathrm{~h}$ post-infection. At least $6 \times 10^{7}$ cells were used for RNA extraction, and all RNA from one group was pooled into one sample. Whole RNA-seq libraries, including lncRNA, circRNA, mRNA, and miRNA, were prepared by Novogene Bioinformatics Technology (Tianjin, China) and sequenced with an Illumina HiSeq 2000/4000 platform (San Diego, CA, USA). Finally, potential BMP9 signaling pathways were identified using KOBAS (2.0) software [57,58], which was used to calculate transcriptome enrichment for mRNA, lncRNA, circRNA, and miRNA. BMP9related KEGG pathways were identified as described[59,60,61].

\section{Statistical analysis}

Data were analyzed by GraphPad Prism 7 and SAS 9. Significant differences between AdBMP9 and Ad-GFP groups were determined by Student's $t$-tests. Hypergeometric P-values < 0.05 were calculated to identify significant associations in KEGG pathways.

\section{Results}

\section{BMP9 upregulates osteogenic differentiation markers in vitro}

We first tested our recombinant adenoviruses and observed that Ad-BMP9 upregulated BMP9 expression (Fig. 1A-B). GFP expression was also present in Ad-BMP9-infected cells 
(Fig. 1A-B). BMP9 is a potent osteogenesis inducer. Therefore, we next tested BMP9 function in GC-1spg cells. Several osteogenic differentiation markers, including Runx2, osteocalcin (OCN), osteopontin (OPN), and Sox9 [17,38,53], were upregulated by BMP9 (Fig. 1C). We then examined the early osteogenic markers ALP $[17,62,63]$ and mineral node formation. ALP activity was analyzed by histochemical staining. Our results showed that BMP9 overexpression increased early osteogenesis differentiation, indicated by ALP activity in GC-1spg cells (Fig. 1D). Alizarin Red S staining was then used to identify mineral nodes. Similarly, BMP9 overexpression visibly increased mineral nodule formation in vitro (Fig. 1E). These results suggest that BMP9 regulates osteogenic differentiation of GC-1spg cells.

Fig 1 Exogenous BMP9 augments osteogenic differentiation marker expression and potentiates osteogenic differentiation [A \& B] Recombinant Ad-BMP9 upregulates BMP9 expression in GC-1spg cells. The recombinant adenoviruses Ad-BMP9 and Ad-GFP effectively transduce GC-1spg cells. GFP was detected $24 \mathrm{~h}$ post-infection. Sub-confluent GC-1spg cells were treated with Ad-BMP9 or Ad-GFP for 24 h, 48 h, or 72 h. Total RNA was isolated and analyzed by real-time PCR. ${ }^{*} \mathrm{P}<0.05$. [C] The recombinant adenovirus AdBMP9 mediates BMP9 overexpression in GC-1spg cells. At the indicated time points, treated cells were analyzed by real-time PCR. Osteogenic markers were upregulated by BMP9. $* \mathrm{P}<0.05$. [D] Cells were analyzed by qualitative histochemical alkaline phosphatase (ALP) staining 3, 5, and 7 days after Ad-BMP9 treatment. BMP9 induces the early osteogenic marker ALP in GC-1spg cells (magnification, 200×). [E] Sub-confluent GC-1spg cells were treated with the indicated adenoviral vectors and cultured in mineralization medium for 7 or 9 days. The cells were fixed and stained with Alizarin Red S. Representative mineral nodule images are shown (magnification, 200×). 


\section{BMP9 induces ectopic bone formation in vivo}

To verify our in vitro results that show exogenous BMP9-induced GC-1spg cell osteogenesis, we performed ectopic ossification experiments. Using our previously established stem cell implantation assay [14,17,38,53], we detected injection-site masses in both BMP9 and GFP groups. Masses in the BMP9 group appeared slightly larger than in the GFP group. Micro-CT analysis also showed differences in tissue volume, bone volume, and bone surface area between Ad-BMP9 and Ad-GFP-injected tumors (Fig. 2 A-B).

Fig 2 BMP9 induces mineralization and maturation of ectopic bone formations [A] Subconfluent GC-1spg cells were treated with Ad-BMP9 or Ad-GFP for $16 \mathrm{~h}$. Treated cells were collected for subcutaneous injections into athymic nude mice. Cells were allowed to grow for 4 weeks. Characteristic masses were retrieved and examined by micro-CT. [B] Injection-site products in the BMP9 group were larger than in the GFP group. Ossification structure volume and surface area of the masses in the BMP9 group were also higher than those of the GFP group. [C] Tumor samples were fixed, decalcified, embedded in paraffin, and stained with H\&E, Masson's trichrome, or alcian blue. Representative images are shown. The AdGFP group exhibited less mineralized, immature chondroid matrix compared to the AdBMP9 group.

Histologic analysis verified BMP9 function in GC-1spg cells. Significant differences were not observed between the two groups using H\&E staining, but Masson's trichrome staining revealed more mature cells with increased mineralization from exogenous BMP9 expression. Analogously, alcian blue staining also showed the accumulation of chondroid 
matrix in BMP9-induced GC-1spg cells (Fig. 2C). Taken together, our data demonstrate that BMP9 induces bone formation in GC-1spg cells.

\section{BMP9 activity is transduced by several cellular pathways}

Several recent studies demonstrated that Notch signaling is required for BMP9regulated bone formation in MSCs $[14,27,48,64]$. However, some studies also demonstrated that BMP9 can induce MSCs to differentiate into various cell types $[22,65,66]$. Moreover, aberrant BMP9 expression might be involved in certain diseases [20,29,34]. These studies suggest that several signaling pathways are involved in BMP9-regulated cell fate or proliferation, but the specific pathways are unclear. We analyzed total RNA, including lncRNA (Fig. 3A), miRNA (Fig. 3B), circRNA (Fig. 3C), and mRNA (Fig. 3D), by RNA-seq followed by KEGG pathway analysis. Our analysis showed that TGF- $\beta$, Notch, MAPK, and Ras signaling pathways mediate BMP9-induced differentiation. This result corroborates previous findings identifying the mechanism of BMP9-induced osteogenic differentiation $[14,27,65,66,67,68]$. Simultaneously, our results also revealed that insulin, PPAR, glutathione metabothyroid hormone, TNF, PI3K-Akt, and several cancer pathways are also involved in BMP9-mediated cellular differentiation. Thus, our findings suggest that BMP9 signaling may be targeted for the treatment for adipogenesis, diabetes, cancer, and certain chronic inflammatory diseases $[17,28,29,69,70]$.

Fig 3 GC-1spg cell signaling pathways induced by BMP9 [A] The top 20 KEGG pathways with differentially expressed lncRNA, [B] miRNA, [C] circRNA, and [D] mRNA are shown. 
Discussion

231

232

233

BMP9, which belongs to the TGF- $\beta$ superfamily, can induce MSCs, such as mouse embryonic fibroblasts (MEFs) or C3H10 cells, to differentiate into bone tissue $[17,22,23,71,72]$. Further, MSC-to-osteoblast transition is driven by BMP9, the most potent osteogenesis-inducing BMP signal in vitro and in vivo [21,22,73]. MSCs originating from the mesoderm can be isolated from different tissues $[5,74,75,76,77]$. Indeed, pluripotent stem cells isolated from testis are similar to MSCs, as demonstrated by gene expression profiling studies $[5,77]$. MSCs can be derived from the testis $[5,8,74]$. Though it is possible to differentiate bone tissue from MSCs, few studies have examined BMP9-induced osteogenesis of testis-derived MSCs.

GC-1spg cells, a mouse spermatogonia-derived cell line, represent the differentiation state of type B spermatogonia or preleptotene spermatocytes [1,37]. Several studies have demonstrated GC-1spg cell plasticity [1,4,78,79]. In our study, we drove BMP9 expression using a recombinant adenovirus. Real-time PCR results confirmed that our adenovirus stably overexpresses BMP9 in GC-1spg cells. Secondly, expression of osteogenic markers, including OPN, OCN, Sox9, and Runx2 [17,38,62,65,71,80], is enhanced by BMP9 in GC1spg cells. Furthermore, ALP and mineralized calcium nodules, early osteogenesis markers, are increased by BMP9 overexpression. Therefore, our in vitro results demonstrate that exogenous BMP9 expression promotes osteogenic differentiation and mineralization in GC1spg cells. To verify our in vitro results, we implanted BMP9-overexpressing cells in mice to induce ectopic ossification. Bony mass formation is augmented by exogenous BMP9 expression. Masson's trichrome staining also showed that BMP9 increased trabeculae thickness, and alcian blue staining demonstrated that elevated BMP9 intensified cartilaginous 
matrix accumulation. Combined with Sox9 expression, we posit that chondrogenic signaling is important for BMP9-induced terminal osteogenic differentiation, although the underlying BMP9-mediated mechanism has not been thoroughly investigated.

BMP9 is one of the most potent osteogenic BMPs both in vitro and in vivo [21,22,25], playing a role in several diseases $[29,35,69,81,82,83]$. Signaling by BMP9 is a synergistic factor regulating the proliferation and migration of specific cell types and stem cell differentiation through extensive crosstalk with several signaling pathways. Though numerous signaling pathways are involved in BMP9-mediated cellular differentiation and proliferation, the cellular transduction mechanism of BMP9 remains unclear. To identify the signaling pathways activated by BMP9, we analyzed total RNA, including lncRNA, mRNA, circRNA, and miRNA, by RNA-seq and KEGG pathway analysis. Mechanistically, the TGF$\beta$ type I receptors ALK1 and ALK2 are required for BMP9-induced differentiation [67]. These receptors mediate nuclear signaling via Smad phosphorylation, suggesting that Smad or TGF- $\beta$ signaling pathways mediate BMP9-induced osteogenesis in GC-1spg cells. Related pathways, including TNF $[84,85]$, MAPK $[86,87]$, Wnt [68], and Notch signaling pathways $[14,27,53]$, may also play a role. In our study, KEGG pathway analysis of differentially expressed lncRNA, mRNA, circRNA, and miRNA shows that BMP9 treatment is involved in the cell cycle, focal adhesion, chemokine signaling, and the ErbB signaling pathway. These pathways are related to the MAPK signaling pathway [88], differentiation and angiogenesis [89], and the PPAR signaling pathway, which is involved in lipid metabolism and adipocyte differentiation [90].

Certain limitations have been identified in our study. MEFs and C3H10 are frequently used in osteogenesis studies, however, GC-1spg cells are rarely used in BMP9 research. This may explain why our results differ from previous findings on BMP9 activity. Indeed, BMP9- 

pathways identified in our study are induced by the adenovirus and not by BMP9 itself. investigating BMP9-mediated bone formation are warranted.

\section{Acknowledgments}

\section{References}

[1] Hofmann MC, Narisawa S, Hess RA, Millán JL. Immortalization of germ cells and somatic testicular cells using the SV40 large T antigen. Exp Cell Res. 1992;201: 417-435.

[2] McLean DJ, Friel PJ, Johnston DS, Griswold MD. Characterization of spermatogonial stem cell maturation and differentiation in neonatal mice. Biol Reprod. 2003;69: 20852091.

[3] Mitsuru K, Takehiko O. Spermatogonial stem cells: Progress and prospects. Asian J Androl. 2015;17: 771-775. 
[4] Hara K, Nakagawa T, Enomoto H, Suzuki M, Yamamoto M, Simons BD, et al. Mouse spermatogenic stem cells continually interconvert between equipotent singly isolated and syncytial states. Cell Stem Cell, 2014;14: 658-672.

[5] De Chiara L, Famulari ES, Fagoonee S, van Daalen SKM, Buttiglieri S, Revelli A, et al. Characterization of human mesenchymal stem cells isolated from the testis. Stem Cells Int. 2018;2018: 4910304.

[6] Seung-Won L, Wu GM, Choi NY, Lee HJ, Bang JS, Lee Y, et al. Self-reprogramming of spermatogonial stem cells into pluripotent stem cells without microenvironment of feeder cells. Mol Cells. 2018;41: 631-638.

[7] Chikhovskaya JV, Jonker MJ, Meissner A, Breit TM, Repping S, van Pelt AM. Human testis-derived embryonic stem cell-like cells are not pluripotent, but possess potential of mesenchymal progenitors. Human Reprod. 2012;27: 210-221.

[8] Guan KM, Karim N, Maier LS, Wagner S, Dressel R, Lee JH, et al. Pluripotency of spermatogonial stem cells from adult mouse testis. Nature, 2006;440: 1199-1203.

[9] Uccelli A, Moretta L, Pistoia V. Mesenchymal stem cells in health and disease. Nature Reviews. Immunology. 2008; 8: 726-736.

[10]Qin YH, Guan JJ, Zhang CQ. Mesenchymal stem cells: mechanisms and role in bone regeneration. Postgrad Med J. 2014;90: 643-647.

[11]Okolicsanyi RK, Camilleri ET, Oikari LE, Yu C, Cook SM, van Wijnen AJ, et al. Human mesenchymal stem cells retain multilineage differentiation capacity including neural marker expression after extended in vitro expansion. PLoS One. 2015;10: $\mathrm{e} 0137255$. 
[12]Augello A, De Bari C. The regulation of differentiation in mesenchymal stem cells. Hum Gene Ther. 2010;21: 1226-1238.

[13]Berendsen AD, Olsen BR. Bone development. Bone. 2015;80: 14-18.

[14]Cui J, Zhang WW, Huang EY, Wang J, Liao J, Li R, et al. BMP-9-induced osteoblastic differentiation requires functional Notch signaling in mesenchymal stem cells. Lab Invest. 2019;99: 58-71.

[15]Luu HH, Song WX, Luo XJ, Manning D, Luo J, Deng ZL, et al. Distinct roles of bone morphogenetic proteins in osteogenic differentiation of mesenchymal stem cells. J Orthop Res. 2007;25: 665-677.

[16]Deng ZL, Sharff KA, Tang N, Song WX, Luo J, Luo X, et al. Regulation of osteogenic differentiation during skeletal development. Front Biosci. 2008;13: 2001-2021.

[17]Wang J, Liao JY, Zhang FG, Song D, Lu M, Liu J, et al. NEL-like molecule-1 (Nell1) is regulated by bone morphogenetic protein 9 (BMP-9) and potentiates BMP-9-induced osteogenic differentiation at the expense of adipogenesis in mesenchymal stem cells. Cell Physiol Biochem. 2017;41: 484-500.

[18]Zhao GQ, Deng K, Labosky PA, Liaw L, Hogan BL. The gene encoding bone morphogenetic protein $8 \mathrm{~B}$ is required for the initiation and maintenance of spermatogenesis in the mouse. Genes Dev. 1996;10: 1657-1669.

[19]Itman C, Loveland KL. SMAD expression in the testis: an insight into BMP regulation of spermatogenesis. Dev Dyn. 2008;237: 97-111.

[20]Wang RN, Green J, Wang Z, Deng Y, Qiao M, Peabody M, et al. Bone morphogenetic protein (BMP) signaling in development and human diseases. Genes Dis. 2014;1: 87-105. 
[21]Kang Q, Song WX, Luo Q, Tang N, Luo J, Luo X, et al. A comprehensive analysis of the dual roles of BMPs in regulating adipogenic and osteogenic differentiation of mesenchymal progenitor cells. Stem Cells Dev. 2009;18: 545-559.

[22]Kang Q, Sun MH, Cheng H, Peng Y, Montag AG, Deyrup AT, et al. Characterization of the distinct orthotopic bone-forming activity of 14 BMPs using recombinant adenovirusmediated gene delivery. Gene Ther. 2004;11: 1312-1320.

[23]Cheng HW, Jiang W, Phillips FM, Haydon RC, Peng Y, Zhou L, et al. Osteogenic activity of the fourteen types of human bone morphogenetic proteins (BMPs). J Bone Joint Surg Am. 2003;85: 1544-1552.

[24]Lamplot JD, Qin JQ, Nan G, Wang J, Liu Xx, Yin L, et al. BMP 9 signaling in stem cell differentiation and osteogenesis. Am J Stem Cells. 2013;2: 1-21.

[25]Mi LZ, Brown CT, Gao Y, Tian Y, Le VQ, Walz T, et al. Structure of bone morphogenetic protein 9 procomplex. Proc Natl Acad Sci USA. 2015;112: 3710-3715.

[26] Song JJ, Celeste AJ, Kong FM, Jirtle RL, Rosen V, Thies RS. Bone morphogenetic protein-9 binds to liver cells and stimulates proliferation. Endocrinology. 1995;136: $4293-4297$.

[27]Cao JJ, Wei YL, Lian J, Yang L, Zhang X, Xie J, et al. Notch signaling pathway promotes osteogenic differentiation of mesenchymal stem cells by enhancing BMP9/Smad signaling. Int J Mol Med. 2017;40: 378-388.

[28]Chen C, Grzegorzewski KJ, Barash S, Zhao Q, Schneider H, Wang Q, et al. An integrated functional genomics screening program reveals a role for BMP-9 in glucose homeostasis. Nat Biotechnol. 2003;21: 294-301. 
[29]Luo Y, Li L, Xu XH, Wu T, Yang M, Zhang C, et al. Decreased circulating BMP-9 levels in patients with Type 2 diabetes is a signature of insulin resistance. Clin Sci. 2017;131: 239-246.

[30]Truksa J, Peng HF, Lee P, Beutler E. Bone morphogenetic proteins 2, 4, and 9 stimulate murine hepcidin 1 expression independently of Hfe, transferrin receptor 2 (Tfr2), and IL6. Proc Natl Acad Sci USA. 2006;103: 10289-11029.

[31]Long L, Ormiston ML, Yang XD, Southwood M, Gräf S, Machado RD, et al. Selective enhancement of endothelial BMPR-II with BMP 9 reverses pulmonary arterial hypertension. Nat Med. 2015;21: 777-785.

[32]Levet S, Ciais D, Merdzhanova G, Mallet C, Zimmers TA, Lee SJ, et al. Bone morphogenetic protein 9 (BMP-9) controls lymphatic vessel maturation and valve formation. Blood. 2013;122: 598-607.

[33] Yoshimatsu Y, Lee YG, Akatsu Y, Taguchi L, Suzuki HI, Cunha SI, et al. Bone morphogenetic protein-9 inhibits lymphatic vessel formation via activin receptor-like kinase 1 during development and cancer progression. Proc Natl Acad Sci USA. 2013;110: 18940-18945.

[34]Wang GL, Fan R, Ji R, Zou W, Penny DJ, Varghese NP, et al. Novel homozygous BMP9 nonsense mutation causes pulmonary arterial hypertension: a case report. BMC Pulm Med. 2016; 16:17.

[35]Addante A, Roncero C, Almalé L, Lazcanoiturburu N, Garcia-Álvaro M, Fernández M, et al. Bone morphogenetic protein 9 as a key regulator of liver progenitor cells in DDCinduced cholestatic liver injury. Liver Int. 2018.38: 1664-1675. 
[36]Li R, Zhang W, Cui J, Shui W, Yin L, Wang Y, Targeting BMP-9-promoted human osteosarcoma growth by inactivation of Notch signaling. Curr Cancer Drug Targets. 2014;14: 274-285.

[37]Lin Y, Liu Z, Liu X, Zhang Y, Rong Z, Li D. Microarray-based analysis of the gene expression profile in GC-1 spg cells transfected with spermatogenesis associated gene 12 . Int J Mol Med. 2013;31: 459-466.

[38]Zhang J, Weng Y, Liu X, Wang J, Zhang W, Kim SH, et al. Endoplasmic reticulum (ER) stress inducible factor cysteine-rich with EGF-like domains 2 (Creld2) is an important mediator of BMP-9-regulated osteogenic differentiation of mesenchymal stem cells. PLoS One. 2013;8: e73086.

[39] Wu N, Zhang H, Deng F, Li R, Zhang W, Chen X, et al. Overexpression of Ad5 precursor terminal protein accelerates recombinant adenovirus packaging and amplification in HEK-293 packaging cells. Gene Ther. 2014;21: 629-637.

[40] Yang Y, Feng Y, Feng X, Liao S, Wang X, Gan H, et al. BMP4 cooperates with retinoic acid to induce the expression of differentiation markers in cultured mouse spermatogonia. Stem Cells Int. 2016;2016: 9536192.

[41]Radhakrishnan K, Bhagya KP, Kumar AT, Devi AN, Sengottaiyan J, Kumar PG. Autoimmune regulator (AIRE) is expressed in spermatogenic cells, and it altered the expression of several nucleic-acid-binding and cytoskeletal proteins in germ cell 1 spermatogonial (GC1-spg) cells. Mol Cell Proteomics. 2016;15: 2686-2698.

[42]He TC, Zhou S, da Costa LT, Yu J, Kinzler KW, Vogelstein B. A simplified system for generating recombinant adenoviruses. Proc Natl Acad Sci USA. 1998;95: 2509-2514. 
407

408

409

410

411

412

413

414

415

416

417

418

419

420

421

422

423

424

425

426

427

[43]Luo J, Deng ZL, Luo X, Tang N, Song WX, Chen J, et al. A protocol for rapid generation of recombinant adenoviruses using the AdEasy system. Nat Protoc. 2007;2: $1236-1247$.

[44]Untergasser A, Cutcutache I, Koressaar T, Ye J, Faircloth BC, Remm M, et al. Primer3-new capabilities and interfaces. Nucleic Acids Res. 2012;40: e115.

[45]Koressaar T, Remm M. Enhancements and modifications of primer design program Primer3. Bioinformatics. 2007;23: 1289-1291.

[46]Livak KJ, Schmittgen TD. Analysis of relative gene expression data using real-time quantitative PCR and the 2(-Delta C(T)) Method. Methods, 2001;25: 402-408.

[47] Schmittgen TD, Livak KJ. Analyzing real-time PCR data by the comparative C(T) method. Nat Protoc. 2008;3: 1101-1108.

[48]Liao J, Yu X, Hu X, Fan J, Wang J, Zhang Z, et al. lncRNA H19 mediates BMP-9induced osteogenic differentiation of mesenchymal stem cells (MSCs) through Notch signaling. Oncotarget, 2017;8: 53581-55360.

[49]Halbhuber KJ, Krieg R, Geidel O, Dietz W. A modified Ce/Mg-BCIP-NBT formazan/indigoblue technique for demonstration of non-specific alkaline phosphatase activity. Cell Mol Biol. 2004;50 Online Pub: OL507-OL514.

[50]Brauer A, Pohlemann T, Metzger W. Osteogenic differentiation of immature osteoblasts: Interplay of cell culture media and supplements. Biotech Histochem. 2016;91: 161-169.

[51]Puchtler H, Meloan SN, Terry MS. On the history and mechanism of alizarin and alizarin red S stains for calcium. J Histochem Cytochem. 1969;17: 110-124. 
[52]Wang N, Zhang WW, Cui J, Zhang H, Chen X, Li R, et al. The piggyBac transposonmediated expression of SV40 T antigen efficiently immortalizes mouse embryonic fibroblasts (MEFs). PLoS One. 2014;9: e97316.

[53]Yan SJ, Zhang RY, Wu K, Cui J, Huang S, Ji X, et al. Characterization of the essential role of bone morphogenetic protein 9 (BMP-9) in osteogenic differentiation of mesenchymal stem cells (MSCs) through RNA interference. Genes Dis. 2018;5: 172-184.

[54]Liu H, Zhong L, Yuan T, Chen S, Zhou Y, An L, et al. MicroRNA-155 inhibits the osteogenic differentiation of mesenchymal stem cells induced by BMP-9 via downregulation of BMP signaling pathway. Int J Mol Med. 2018;41: 3379-3393.

[55] Song JW, Cha JY, Bechtold TE, Park YC. Influence of peri-implant artifacts on bone morphometric analysis with micro-computed tomography. Int J Oral Max Impl. 2013;28: $519-525$.

[56] Waarsing JH, Day JS, van der Linden JC, Ederveen AG, Spanjers C, De Clerck N, et al. Detecting and tracking local changes in the tibiae of individual rats: a novel method to analyse longitudinal in vivo micro-CT data. Bone. 2004;34: 163-169.

[57]Mao X, Cai T, Olyarchuk JG, Wei L. Automated genome annotation and pathway identification using the KEGG Orthology $(\mathrm{KO})$ as a controlled vocabulary. Bioinformatics. 2005;21: 3787-3793.

[58]Wu J, Mao X, Cai T, Luo J, Wei L. KOBAS server: a web-based platform for automated annotation and pathway identification. Nucleic Acids Res. 2006;34: W720-W724.

[59]Zhang S, Zhu D, Li H, Li H, Feng C, Zhang W. Characterization of circRNA-associatedceRNA networks in a senescence-accelerated mouse prone 8 brain. Mol Ther. 2017;25: 2053-2061. 
[60]Zhang S, Qin C, Cao G, Xin W, Feng C, Zhang W. Systematic analysis of long noncoding RNAs in the senescence-accelerated mouse prone 8 brain using RNA sequencing. Mol Ther Nucleic Acids. 2016;5: e343.

[61]Wu Y, Zhang Y, Niu M, Shi Y, Liu H, Yang D. et al. Whole-transcriptome analysis of CD133+CD144+ cancer stem cells derived from human laryngeal squamous cell carcinoma cells. Cell Physiol Biochem. 2018;47: 1696-1710.

[62]Ching HS, Luddin N, Rahman IA, Ponnurag KT. Expression of odontogenic and osteogenic markers in DPSCs and SHED: A Review. Curr Stem Cell Res Ther. 2017;12: 71-79.

[63]Pujari-Palmer M, Pujari-Palmer S, Lu X, Lind T, Melhus H, Engstrand T, et al. Pyrophosphate stimulates differentiation, matrix gene expression and alkaline phosphatase activity in osteoblasts. PLoS One. 2016;11: e0163530.

[64]Liu P, Man YQ, Wang Y, Bao Y. Mechanism of BMP-9 promotes growth of osteosarcoma mediated by the Notch signaling pathway. Oncol Lett. 2016;11: 1367-1370.

[65] Wang P, Wang Y, Tang W, Wang X, Pang Y, Yang S, et al. Bone morphogenetic protein-9 enhances osteogenic differentiation of human periodontal ligament stem cells via the JNK pathway. PLoS One. 2017;12: e0169123.

[66]Furue K, Sena K, Sakoda K, Nakamura T, Noguchi K. Involvement of the phosphoinositide 3-kinase/Akt signaling pathway in bone morphogenetic protein 9stimulated osteogenic differentiation and stromal cell-derived factor 1 production in human periodontal ligament fibroblasts. Eur J Oral Sci. 2017;125: 119-126. 
472

473

474

475

476

477

478

479

480

481

482

483

484

485

486

487

488

489

490

491

492

493

494

[67]Luo J, Tang M, Huang J, He BC, Gao JL, Chen L, et al. TGFbeta/BMP type I receptors ALK1 and ALK2 are essential for BMP-9-induced osteogenic signaling in mesenchymal stem cells. J Cell Biochem. 2010;285: 29588-29598.

[68]Zhu JH, Liao YP, Li FS, Hu Y, Li Q, Ma Y, et al. Wnt11 promotes BMP-9-induced osteogenic differentiation through BMPs/Smads and p38 MAPK in mesenchymal stem cells. J Cell Biochem. 2018;119: 9462-9473.

[69]Li S, Dai H, He Y, Peng S, Zhu T, Wu Y, et al. BMP-9 inhibits the growth of breast cancer cells by downregulation of the PI3K/Akt signaling pathway. Oncol Rep. 2018;40: $1743-1751$.

[70]Liu Y, Liu YZ, Zhang R, Wang X, Huang F, Yan Z, et al. All-trans retinoic acid modulates bone morphogenic protein 9-induced osteogenesis and adipogenesis of preadipocytes through BMP/Smad and $\mathrm{Wnt} / \beta$-catenin signaling pathways. The Int $\mathbf{J}$ Biochem Cell Biol. 2014;47: 47-56.

[71]Wang X, Huang J, Huang F, Zong JC, Tang X, Liu Y, et al. Bone morphogenetic protein 9 stimulates callus formation in osteoporotic rats during fracture healing. Mol Med Rep. 2017;15: 2537-2545.

[72]Fujioka-Kobayashi M, Sawada K, Kobayashi E, Schaller B, Zhang Y, Miron RJ. Recombinant human bone morphogenetic protein 9 (rhBMP-9) induced osteoblastic behavior on a collagen membrane compared with rhBMP2. J Periodontol. 2016;87: e101-e107.

[73]Khorsand B, Elangovan S, Hong L, Dewerth A, Kormann MS, Salem AK. A comparative study of the bone regenerative effect of chemically modified RNA encoding BMP-2 or BMP-9. AAPS J. 2017;19: 438-446. 
[74]Bai Y, Zhu C, Feng M, Wei H, Li L, Tian X, et al. Previously claimed male germline stem cells from porcine testis are actually progenitor Leydig cells. Stem Cell Res Ther. 2018;9: 200.

[75] Vangsness CT Jr, Sternberg H, Harris L. Umbilical cord tissue offers the greatest number of harvestable mesenchymal stem cells for research and clinical application: a literature review of different harvest sites. Arthroscopy. 2015;31: 1836-1843.

[76]Cordeiro-Spinetti E, de Mello W, Trindade LS, Taub DD, Taichman RS, Balduino A. Human bone marrow mesenchymal progenitors: perspectives on an optimized in vitro manipulation. Front Cell Dev Biol. 2014;2: 7.

[77]Gonzalez R, Griparic L, Vargas V, Burgee K, Santacruz P, Anderson R, et al. A putative mesenchymal stem cells population isolated from adult human testes. Biochem Biophys Res Commun. 2009;385: 570-575.

[78]Cooke PS, Simon L, Nanjappa MK, Medrano TI, Berry SE. Plasticity of spermatogonial stem cells. Asian J Androl. 2015; 17: 355-359.

[79]Chandran U, Indu S, Kumar AT, Devi AN, Khan I, Srivastava D, et al. Expression of Cnnm1 and its association with stemness, cell cycle, and differentiation in spermatogenic cells in mouse testis. Biol Reprod. 2016;95: 7.

[80]Loebel C, Czekanska EM, Bruderer M, Salzmann G, Alini M, Stoddart MJ. In vitro osteogenic potential of human mesenchymal stem cells is predicted by Runx2/Sox 9 ratio. Tissue Eng Part A. 2015;21: 115-123.

[81]Nikolic I, Yung LM, Yang P, Malhotra R, Paskin-Flerlage SD, Dinter T, et al. Bone morphogenetic protein 9 is a mechanistic biomarker of portopulmonary hypertension. Am J Respir Crit Care Med. 2019;199: 891-902. 
[82] Gou LY, Liu MY, Xia J, Wan Q, Jiang Y, Sun S, et al. BMP 9 promotes the proliferation and migration of bladder cancer cells through up-regulating lncRNA UCA1. Int J Mol Sci. 2018;19: E1116.

[83] Morine KJ, Qiao X, York S, Natov PS, Paruchuri V, et al. Bone morphogenetic protein 9 reduces cardiac fibrosis and improves cardiac function in heart failure. Circulation. 2018;138: 513-526.

[84] Szondy Z, Pallai A. Transmembrane TNF-alpha reverse signaling leading to TGF-beta production is selectively activated by TNF targeting molecules: Therapeutic implications. Pharmacol Res. 2017;115: 124-132.

[85]Qadir AS, Lee HL, Baek KH, Park HJ, Woo KM, Ryoo HM, et al. Msx2 is required for TNF- a -induced canonical Wnt signaling in 3T3-L1 preadipocytes. Biochem Biophys Res Commun. 2011;408: 399-404.

[86]Sun Y, Liu W Z, Liu T, Feng X, Yang N, Zhou HF. Signaling pathway of MAPK/ERK in cell proliferation, differentiation, migration, senescence and apoptosis. J Recept Signal Transduction Res. 2015;35: 600-604.

[87]An N, Peng J, He G, Fan X, Li F, Chen H. Involvement of activation of mitogenactivated protein kinase (MAPK)/extracellular signal-regulated kinase (ERK) signaling pathway in proliferation of urethral plate fibroblasts in finasteride-induced rat hypospadias. Med. Sci. Monit. 2018.24: 8984-8992.

[88]Cronise KE, Hernandez BG, Gustafson DL, Duval DL. Identifying the ErbB/MAPK signaling cascade as a therapeutic target in canine bladder cancer. Mol Pharmacol. 2019;96: 36-46. 
540 [89]Yang Y, Li B, Zhang X, Zhao Q, Lou X. The zinc finger protein Zfpm1 modulates

541 ventricular trabeculation through neuregulin-ErbB signalling. Dev Biol. 2019;446: 142-

$542 \quad 150$

543 [90]Li Y, Jin D, Xie W, Wen L, Chen W, Xu J. PPAR- $\gamma$ and Wnt regulate the

544 differentiation of MSCs into adipocytes and osteoblasts respectively. Curr Stem Cell Res

545 Ther. 2018;13: 185-192.

546

547 
A

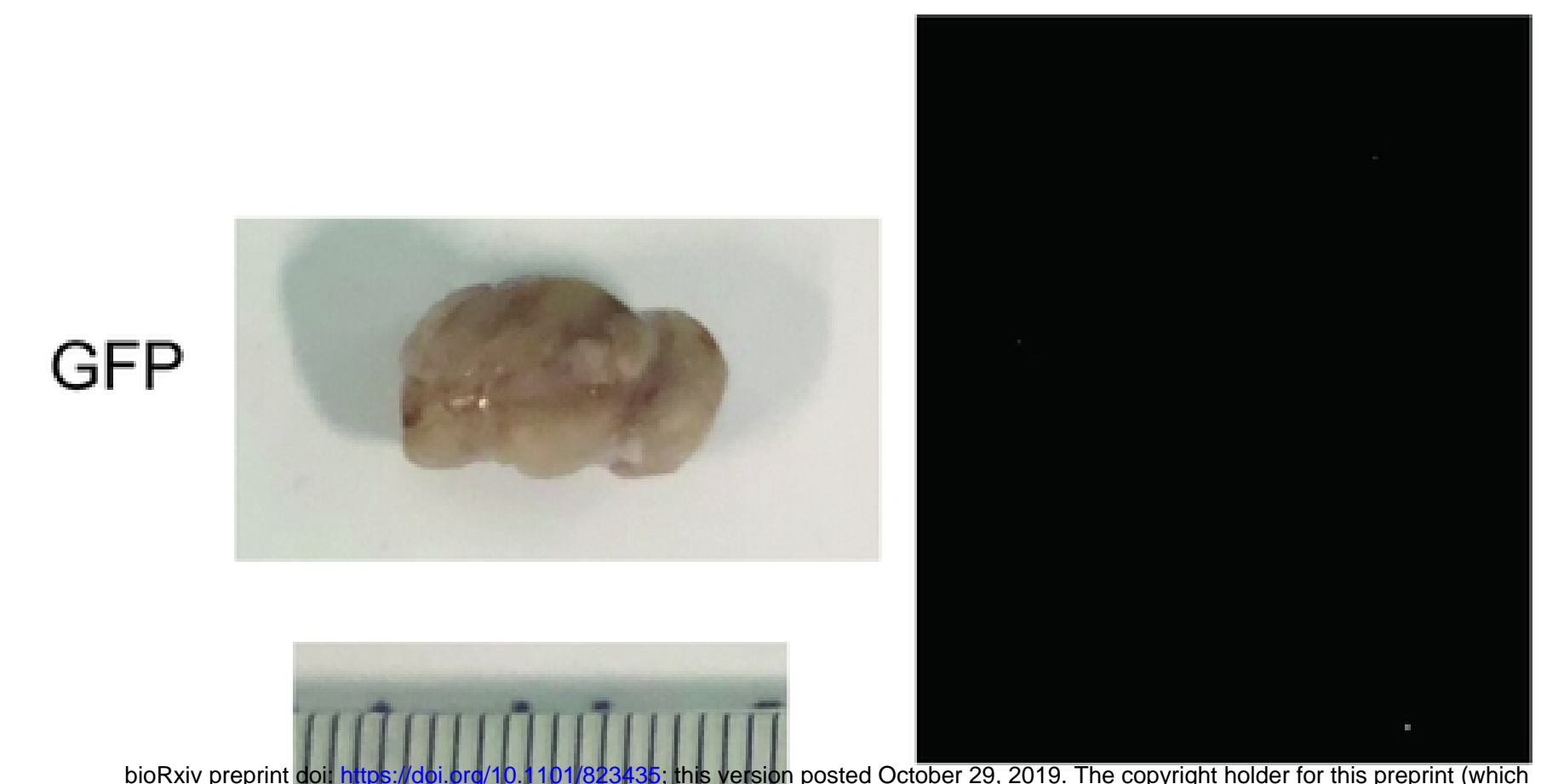

bioRxiv preprint doi: https://doi.org/10.1101/823435; this version posted October 29, 2019. The copyright holder for this preprint (which

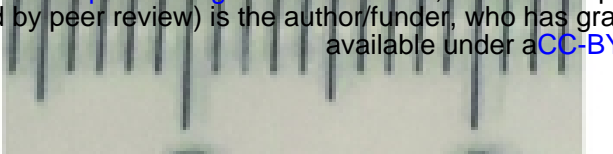
5 6

\section{BMP9}

C

GFP

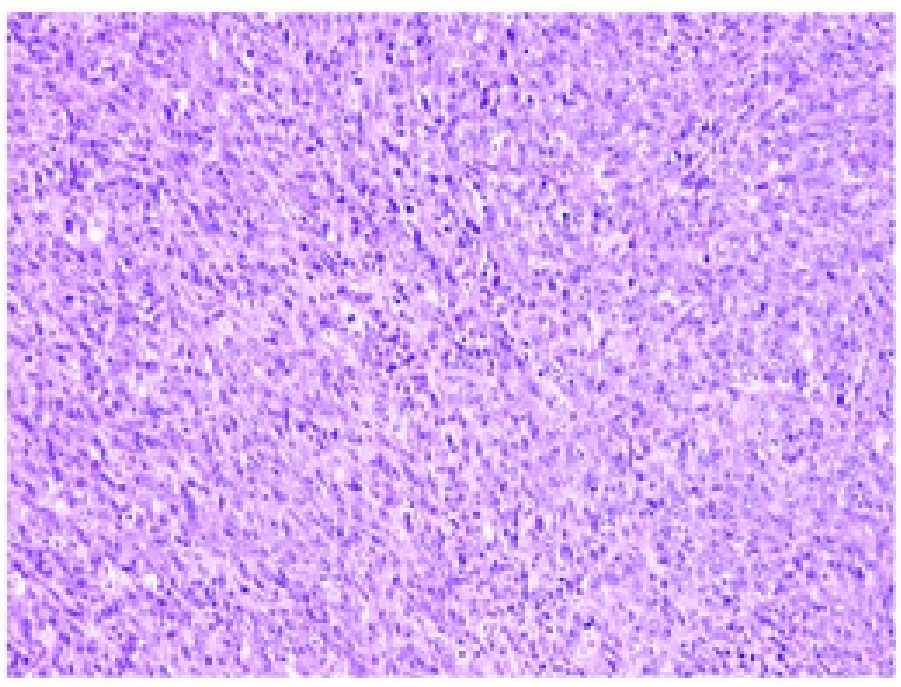

\section{BMP9}

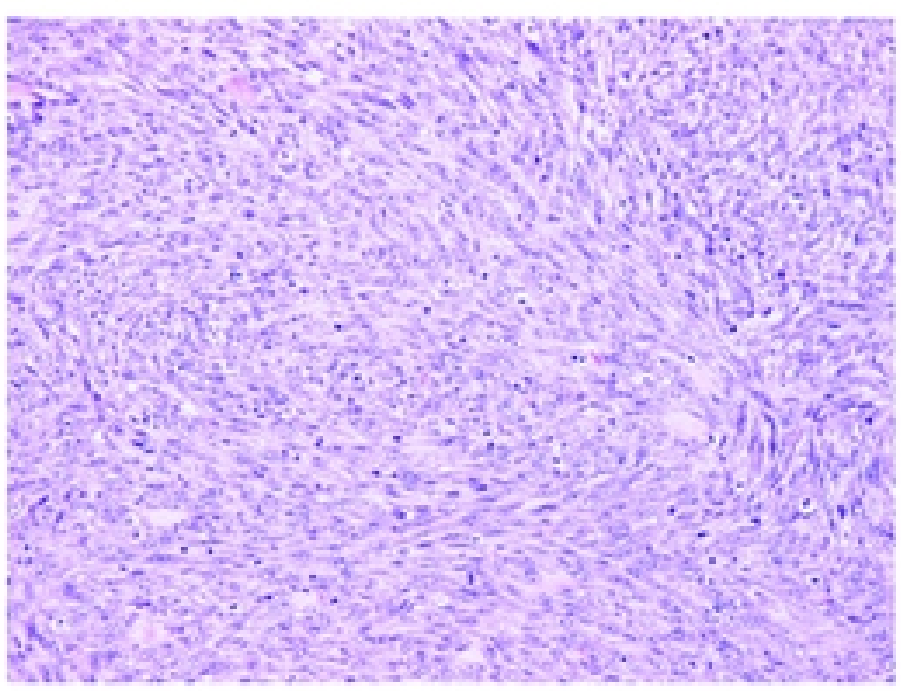

$H$ \& E stain

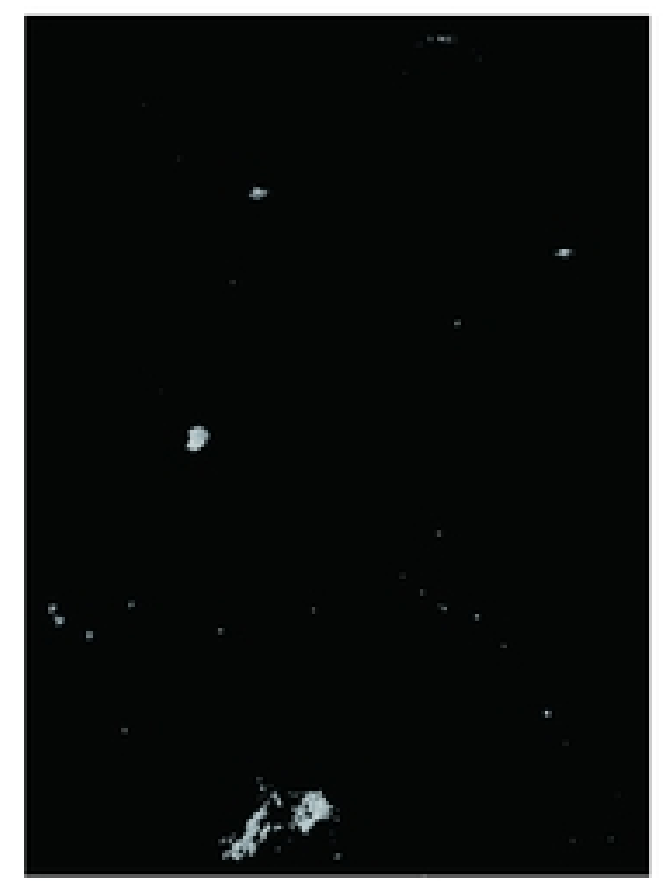

microCT
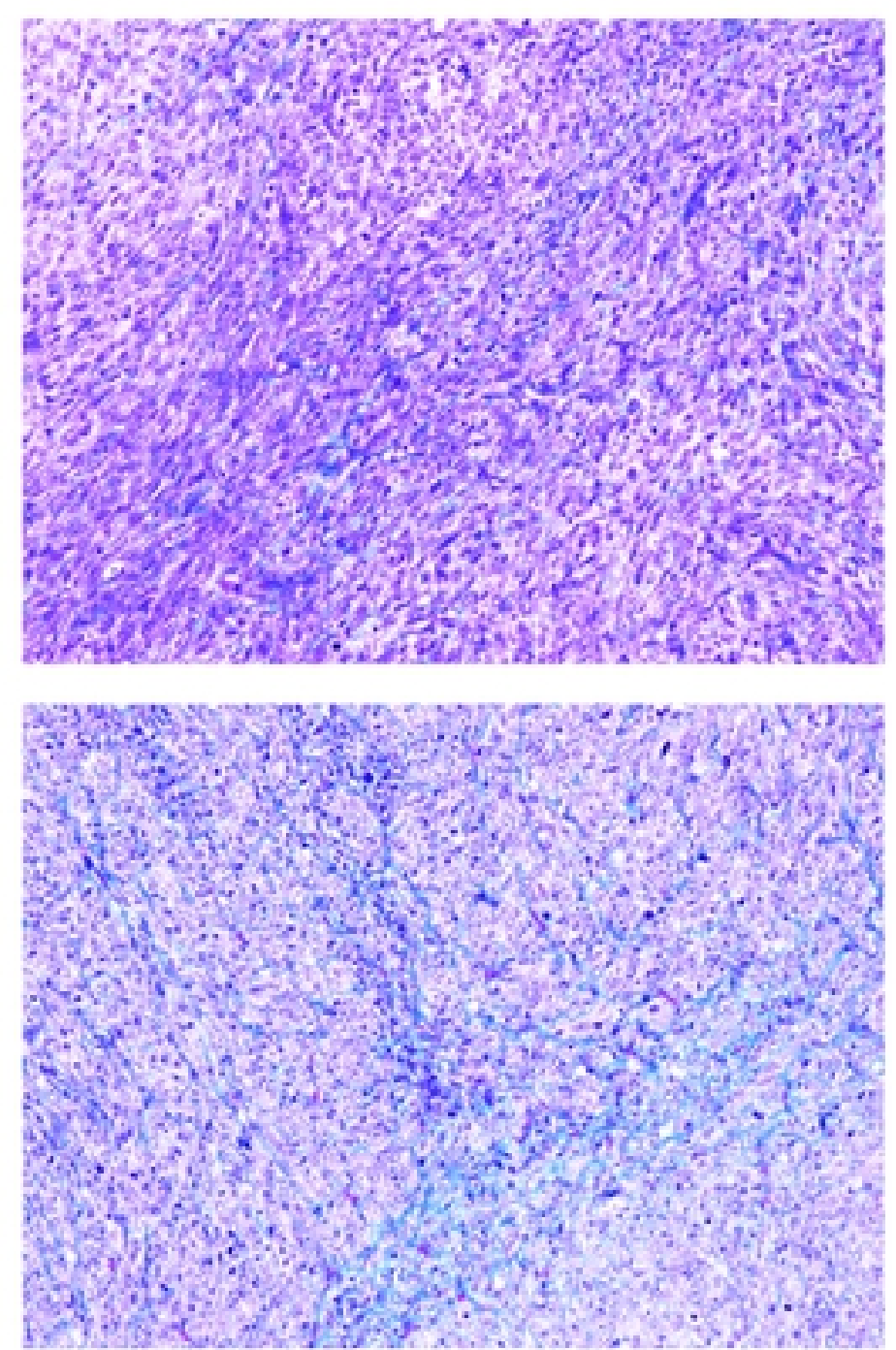

trichrome stain
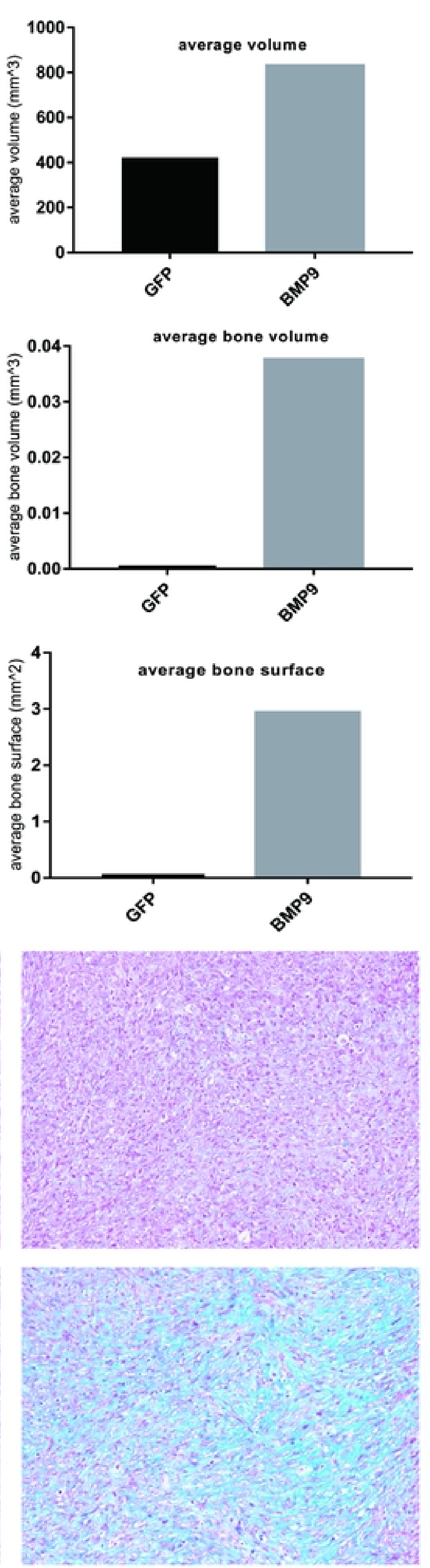

alcian blue stain

\section{Figure2}



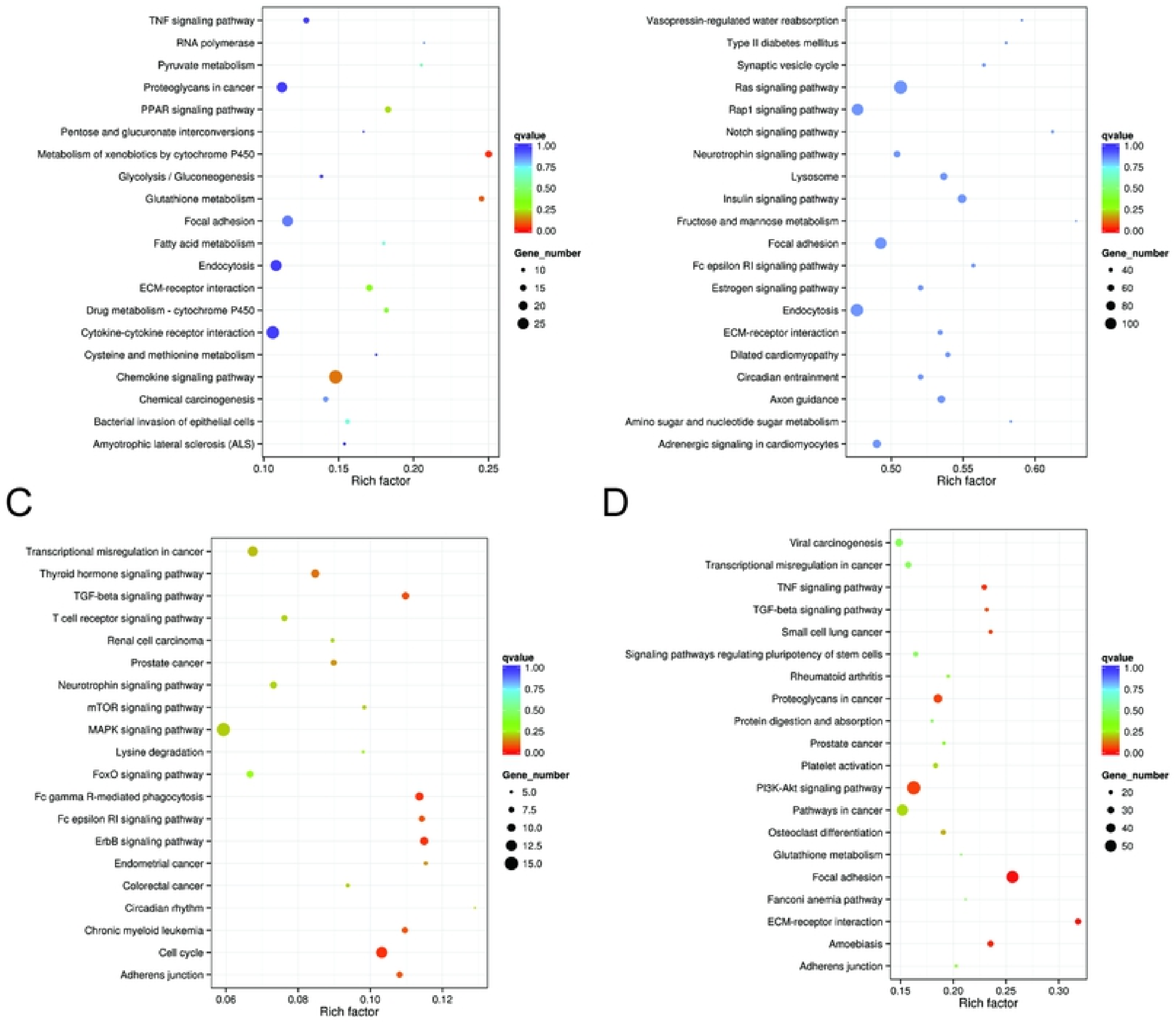

Figure3 